\title{
Further Research on an Anti-Swing Control System for Overhead Cranes
}

\author{
Bronislovas Spruogis \\ Dpt of Mobile Machinery and Railway \\ Transport \\ Vilnius Gediminas Technical University \\ Vilnius, Lithuania \\ bronislovas.spruogis@vgtu.lt
}

\author{
Arunas Jakstas \\ Dpt of Mechanical and Material \\ Engineering \\ Vilnius Gediminas Technical University \\ Vilnius, Lithuania \\ arunas.jakstas@vgtu.lt
}

\author{
Vladimir Gican \\ Dpt of Mechatronics, Robotics and \\ Digital Manufacturing \\ Vilnius Gediminas Technical University \\ Vilnius, Lithuania \\ vladimir.gican@vgtu.lt
}

\author{
Vytautas Turla \\ Dpt of Mechatronics, Robotics and Digital Manufacturing \\ Vilnius Gediminas Technical University \\ Vilnius, Lithuania \\ vytautas.turla@vgtu.lt
}

\author{
Vadim Moksin \\ Dpt of Mechanical and Material Engineering \\ Vilnius Gediminas Technical University \\ Vilnius, Lithuania \\ vadim.moksin@vgtu.lt
}

\begin{abstract}
A method of reducing load oscillations that occur when overhead crane reaches destination position is presented in the article. The use of control drive scheme of crane bridge and trolley that ensures a smooth phase trajectory transition of the load to the optimum trajectory in accordance with Pontryagin's maximum principle is proposed. Mentioned control system changes the magnitude or direction of the traction force at the moment when the load is located above the destination. It is found that the degree of change of the traction force depends on the hoisting rope deviation angle from vertical. This study was conducted in order to provide more accurate and fast handling of loads by overhead crane.
\end{abstract}

Keywords-anti-swing control system; load; oscillations; overhead crane; Pontryagin's maximum principle

\section{INTRODUCTION}

Overhead cranes are widely used in industrial environments to transport loads from one place to another. Due to high safety standards and requirements of small swing angle, high positioning accuracy and short transportation time, crane operation is complicated work that should be automated. However, in industrial practice, most of these machines are manually operated by long-time trained operators. This situation results in low labor productivity, low work safety etc [1]. For this reason, some researchers have started to address the control problem for overhead cranes [2]. A new fuzzy controller for anti-swing and position control of an overhead travelling crane was proposed based on the single input rule modules (SIRMs) dynamically connected fuzzy inference model [3]. Velocity and position of the trolley as well as an angular velocity and swing angle of the rope were selected as inputs while the acceleration of the trolley was selected as an output [3]. If the initial conditions aren't zeroed or disturbances appear during the movement, then closed control systems should be used $[4,5]$. In cases when it is necessary to control the slewing motion in cranes, the systems controlling the angular velocity of the jib can be used. The angular acceleration profile of the jib is considered as an output of these systems [6].

A nonlinear controller was proposed in [7] for the trolley cranes using Lyapunov method. A modified version of slidingsurface control was then utilized to achieve position control of the trolley [7]. A fuzzy logic control system with sliding mode control concept was developed for an overhead crane system in [8]. Authors in [9] suggested nonlinear coupling control law to stabilize overhead crane with three degree of freedom using LaSalle's invariance theorem. In [10] a nonlinear tracking controller for controlling the load velocity and position was designed with two loops: an outer loop was intended for position tracking, and an inner loop was intended for stabilizing the oscillations using a singular perturbation design. However, the desired result was obtained only for the case when the swing angle dynamics is much faster than the trolley motion dynamics. In [11] a nonlinear control scheme incorporating parameter adaptive mechanism was proposed to ensure the overall closed-loop system stability. By applying the designed controller, the position error is driven to zero while the swing angle is rapidly damped to achieve swing stabilization.

It is known that certain types of load lifting mechanisms result in double-pendulum dynamics [12]. Many researchers have worked to provide solutions to the problems posed by the double-pendulum dynamics [13]. Their works can roughly be divided into two categories: feedback control and input shaping. Feedback control strategy uses measurements and estimation of system state to suppress oscillations [12, 14, 15]. In [13] a command-smoothing scheme was presented to suppress the complex oscillations of the load. Simulations of a large range of motions were used to analyze the dynamic 
behavior of the cranes and the robustness of this method. In order to meet the demand for faster load handling, crane motion must be controlled so that its dynamic performance is optimized. Specifically, the control of overhead crane systems aims to achieve both position and anti-swing control [16]. As the swing of the load depends on the acceleration of the trolley, minimizing both operation time and load swing can be defined as partially contradictory requirements. The anti-swing control problem involves reducing the load swing while moving it to the desired position as fast as possible [17]. One particular and effective feedforward approach to reduce vibrations in machines is input shaping [18]. In order to control mechanical systems, a novel fast control strategy was proposed in [19]. The controller includes proportional derivative (PD) regulator and fuzzy cerebellar model articulation controller. For an overhead crane, this control system can realize both position tracking and anti-swing control [19]. In [20], evolutionary-based algorithm for fuzzy logic-based data-driven predictive model of time between failures and adaptive crane control system design were proposed.

In the present paper, possibilities of the optimization of overhead crane control to increase the load positioning accuracy and reduce the transportation time are discussed.

\section{MODEL AND SIMULATION}

Simulation model of the overhead crane was developed using MATLAB/Simulink software [21]. Previous research works [22] showed that significant load swing occurs during transportation by the overhead crane. The swing has negative effect on the performance of the equipment and usually it is required to reduce the load swing amplitude to the admissible level. In order to reduce the load swing amplitude, following measures can be applied:

- Equipment that applies a force to the load at certain moments of time. The direction of such a force is opposite to the direction of deviation of the load.

- A stabilizing force can be also applied to the load attachment point by means of special control of the crane's motors.

It is interesting to compare both control approaches.

The dynamic model of the overhead crane is presented in Figure 1. The crane consists of three parts: a load lifting mechanism, a trolley and a bridge.

The position of the mass $M_{\Theta}$ (Figure 1) can be described by the following formulas:

$$
\begin{aligned}
& x_{m}=L \sin \Theta_{x} \cos \Theta_{y}, \\
& y_{m}=L \sin \Theta_{y},
\end{aligned}
$$

where $L$ is the length of the rope:

$$
L=L_{0}+\delta_{L},
$$

where $L_{0}$ is the length of the rope without a load attached which is determined by angular position of the rope drum, $\delta_{L}$ is the elongation of the rope caused by the load. The deviation angle of the rope (Figure 1) is:

$$
\Theta=\frac{\sqrt{x_{m}^{2}+y_{m}^{2}}}{L} .
$$

Vertical displacement of the load (Figure 1) caused by swing:

$$
z_{m}=L\left(1-\cos \Theta_{x} \cos \Theta_{y}\right)
$$

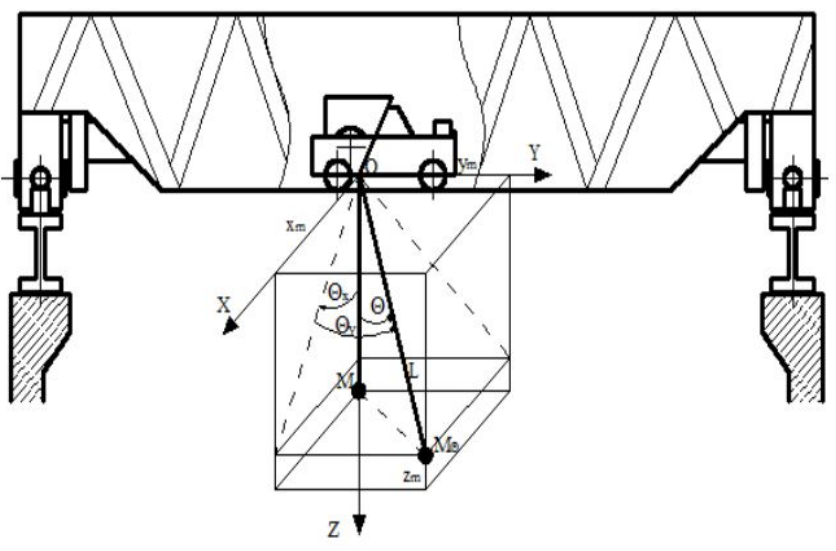

Fig. 1. The dynamic model of the overhead crane

Equations (1-5) are used to determine kinetic and potential energy of the mechanical system of load lifting mechanism as well as its dissipative function in Lagrange equations of second kind. During the creation of the mathematical model, experience of industrial enterprise Vilniaus kranai $A B$ was evaluated claiming that rope angular deviation from the vertical does not exceed 5 degrees. Obtained after appropriate processing in accordance with techniques presented in [23] equations and used coordinates are presented in [21]. Modeling of the mechanical system was carried out with the following parameters (data obtained from the experiments carried out at Vilniaus kranai $\mathrm{AB}$ ): standard gravity $g=9.81 \mathrm{~m} / \mathrm{s}^{2}$, load mass $M=1000 \mathrm{~kg}$, trolley mass $M_{x}=5000 \mathrm{~kg}$, mass of the trolley and the bridge $M_{y}=10000 \mathrm{~kg}$, damping coefficient at the rope attachment point $H_{2}=1 \mathrm{Nms} / \mathrm{rad}$, rope damping coefficient $H_{1}=1000 \mathrm{Ns} / \mathrm{m}$, initial rope tension stiffness $C_{1}=1716000 \mathrm{~N} / \mathrm{m}$, damping coefficient of the trolley along axis $X H_{x}=1000 \mathrm{Ns} / \mathrm{m}$, damping of the crane along axis $Y H_{y}=2000 \mathrm{Ns} / \mathrm{m}$. Nonlinearity of the rope elongation-load relationship is taken into consideration in the mathematical model. For description simplicity, two additional coordinates and their derivatives were introduced:

$$
\begin{aligned}
& X_{L}=L_{0} \Theta_{x}, \quad \dot{X}_{L}=L_{0} \dot{\Theta}_{x}, \\
& X_{M}=X_{0}+X_{L}, \\
& \dot{X}_{M}=\dot{X}_{0}+\dot{X}_{L}, \\
& Y_{L}=L_{0} \Theta_{y}, \quad \dot{Y}_{L}=L_{0} \dot{\Theta}_{y},
\end{aligned}
$$




$$
Y_{M}=Y_{0}+Y_{L}, \quad \dot{Y}_{M}=\dot{Y}_{0}+\dot{Y}_{L}
$$

Obtained model describes the mechanical system of the overhead crane and the load position changes caused by the rope attachment point position changes in directions $X$ and $Y$ and by inclination of the load on the rope by angles $\Theta_{x}$ and $\Theta_{y}$ (Figure 1). Forces $F_{x}$ and $F_{y}$ are generated by the motors of the trolley and the bridge and determine the rope movement attachment point. Motors are controlled by controllers. The algorithm of the controllers can be based on Pontryagin's maximum principle [24]. If the trolley moves with low energy dissipation and in absence of restoring force (which cannot exceed the value predetermined by the motor power), the optimum phase trajectory consists of two parabolas. In the first segment (the acceleration) the control parameter $u \equiv 1$ and the phase trajectory is described by the following equation:

$$
x=\frac{1}{2}(d x)^{2}+s
$$

where $s$ is the arbitrary constant. In the second segment (deceleration) the control parameter $u \equiv-1$ (i.e. the motor is reversed) and the phase point moves to the parabola that crosses the zero point of coordinates:

$$
x=-\frac{1}{2}(d x)^{2}+s
$$

The optimum phase trajectory under absence of restoring force and energy dissipation conditions is presented in Figure 2.

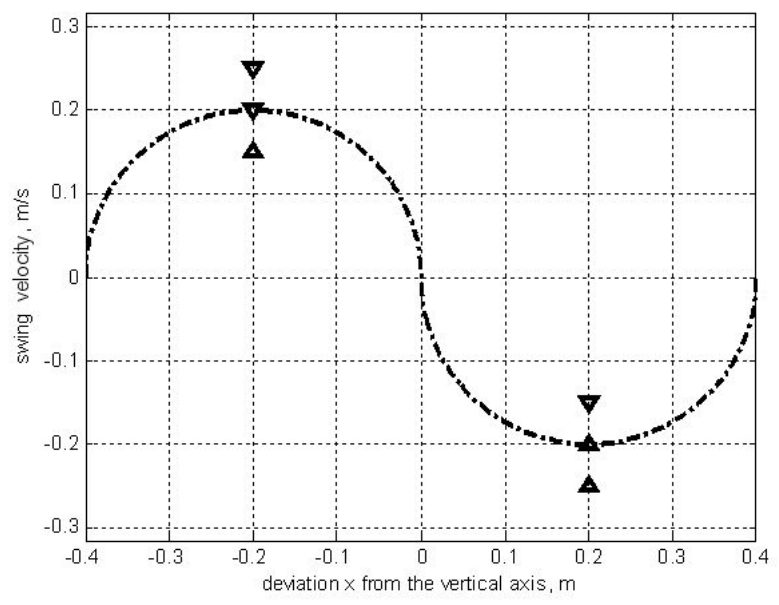

Fig. 2. Optimum phase trajectory in accordance with Pontryagin's maximum principle

In order to investigate the dynamic behavior of mechanical system of the crane, a Simulink diagram was developed. The control subsystem is described in [21]. The model operates as follows. Traction force is applied from the motor of the bridge (entry $F_{x}$ ) to the model and movement of the trolley along the $X$ axis towards the point of coordinate start. During the periods of acceleration and deceleration, the phase trajectories are described by (10) and (11) for the case of negligible dissipative forces. If energy dissipation is significant, acceleration and deceleration curves are different from parabolas. During the Simulink model creation it was assumed that in the case of motor power disconnection, corresponding wheels are braked and then disengaged when the motor is turned on.

Figure 3(a) shows the traction force $F_{x}$ versus time relationship for the case of simple drive braking. Load oscillation amplitude versus time plot obtained during vertical hoisting of the load is presented in Figure 3(b). The traction force was considered to change at the moment when the point of winding of the rope on the drum is located over the place of destination of the load. Phase trajectory of the load is presented in Figure 3(c). An increase of oscillation amplitude is observed during of the braking of the drive (Figure 3(b)). In the case of application of the proposed anti-swing control system, phase trajectory of the load should coincide with the optimum trajectory in accordance with Pontryagin's maximum principle (Figure 2).

It is established that the decrease of traction force from 1300 to $1200 \mathrm{~N}$ eliminates an increase of load oscillation amplitude that occurs due to the change of the traction force (Figures 4(a), 4(b)). However, the oscillations excited by the load movement remain for considerable time (Figure 4(c)). A more detailed study of the load dynamics showed the influence of oscillation phase at the moment of change of traction force. It can be stated that in the case of slight deviation of the rope from vertical position, smooth transition to the optimum phase trajectory is carried out with a significant reduction of traction force while its application direction is maintained (Figure 5). Effective oscillation damping can be achieved also by reversing the traction force (Figures 6(a), 6(b)). In this case, the amount of force that is necessary to apply in the opposite direction is only part of the initial value (Figure 6(a)). A smooth transition to the optimum phase trajectory is achieved as well (Figure 6(c)). In the case of a significant deviation of the rope from vertical position at the moment of change of the traction force (Figure 7) timely transition of the phase trajectory to optimum trajectory is provided by the increase of the traction force while its application direction is maintained.

\section{CONCLUSIONS}

Studies have shown that the application of Pontryagin's maximum principle allows the developing of overhead crane drive control system capable of providing effective rope oscillation damping. In order to simplify the control system, magnitude and direction of the traction force should be changed at the moment when the point of winding of the rope on the drum is located over the place of destination of the load. The change degree of magnitude and direction of the traction force depends on the rope deviation angle from vertical. In the case of slight rope deviation from the vertical position at the moment of change of the traction force, load oscillations are reduced by decreasing the traction force while its application direction is maintained. However, if it is necessary to reduce 
the damping time to a minimum, direction of the traction force should be changed while at the same time its magnitude should be reduced. If at the moment of change of the traction force rope deviation is close to the amplitude of the oscillations, the effective oscillation damping is provided by increasing the traction force while its application direction is maintained. The proposed control system is capable to control both trolley and bridge drives.
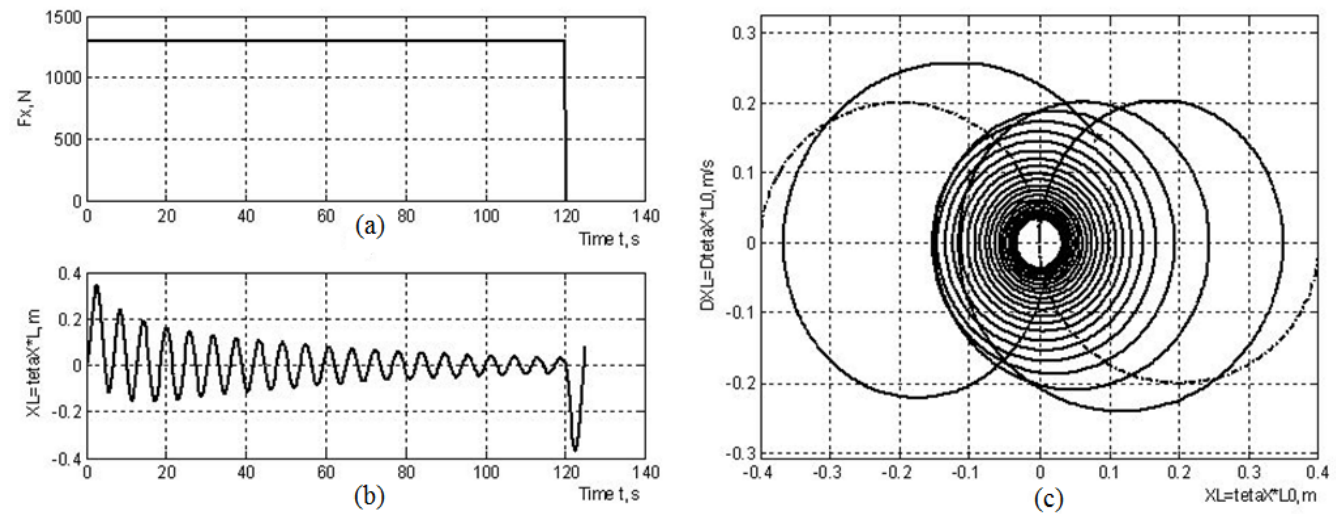

Fig. 3. Traction force (a) and load oscillation amplitude (b) vs. time plots and phase trajectory plot (c) obtained for the case of absence of anti-swing system
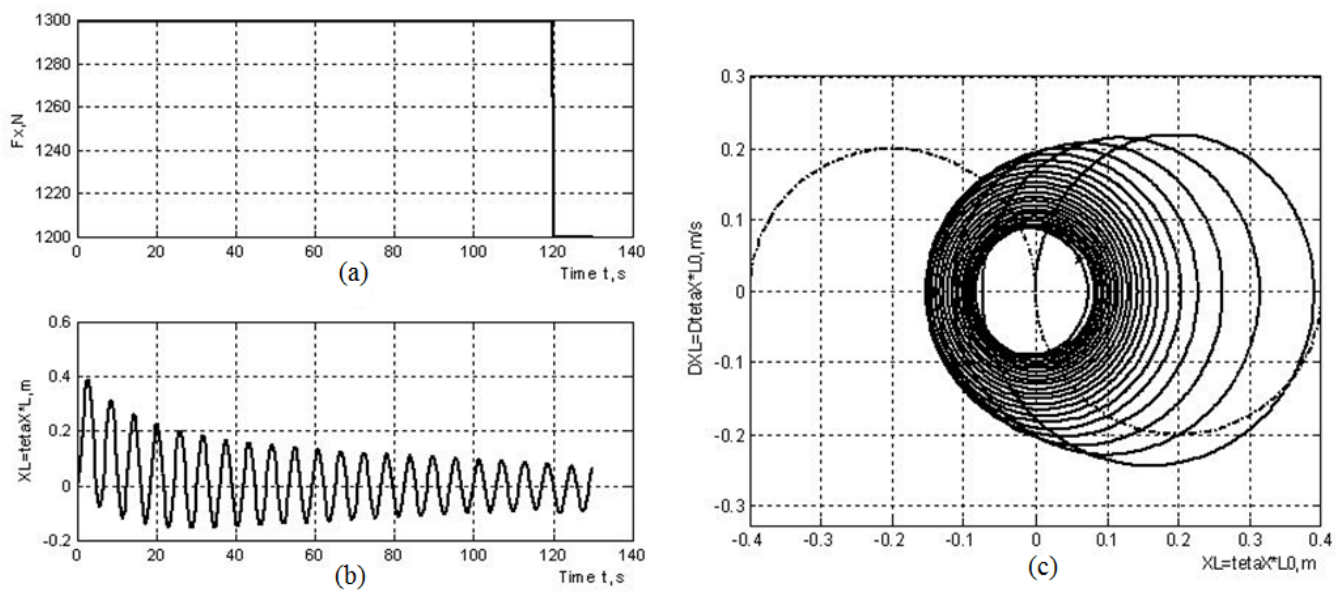

Fig. 4. Traction force (a) and load oscillation amplitude (b) vs. time plots and phase trajectory plot (c) obtained for the case of the reduction of traction force from 1300 to $1200 \mathrm{~N}$

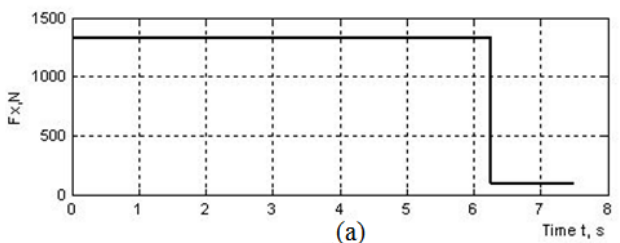

(a)

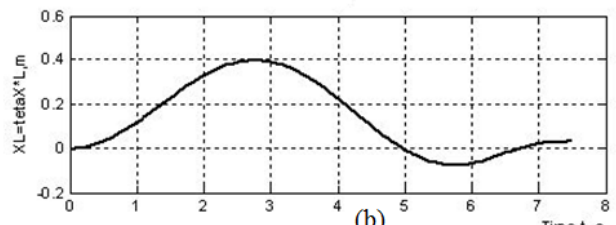

(b)

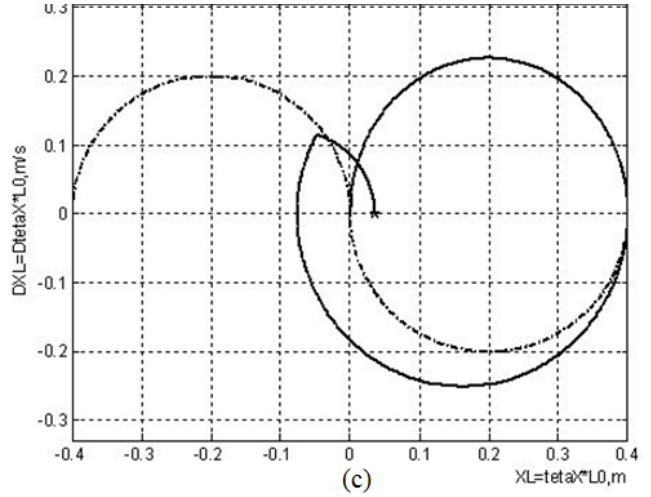

(c)

Fig. 5. Traction force (a) and load oscillation amplitude (b) vs. time plots and phase trajectory plot (c) obtained for the case of the slight deviation of the rope from vertical position at the moment of change of the traction force 

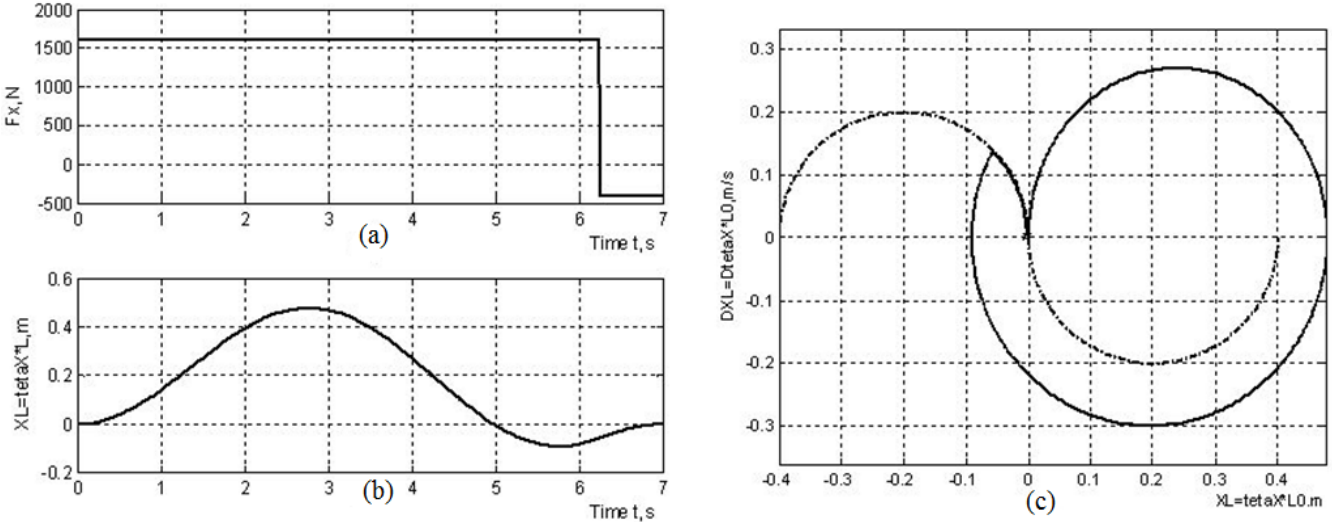

Fig. 6. Traction force (a) and load oscillation amplitude (b) vs. time plots and phase trajectory plot (c) obtained for the case of the reversing of the traction force

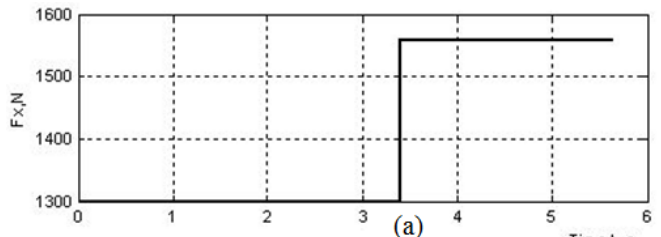

(a)
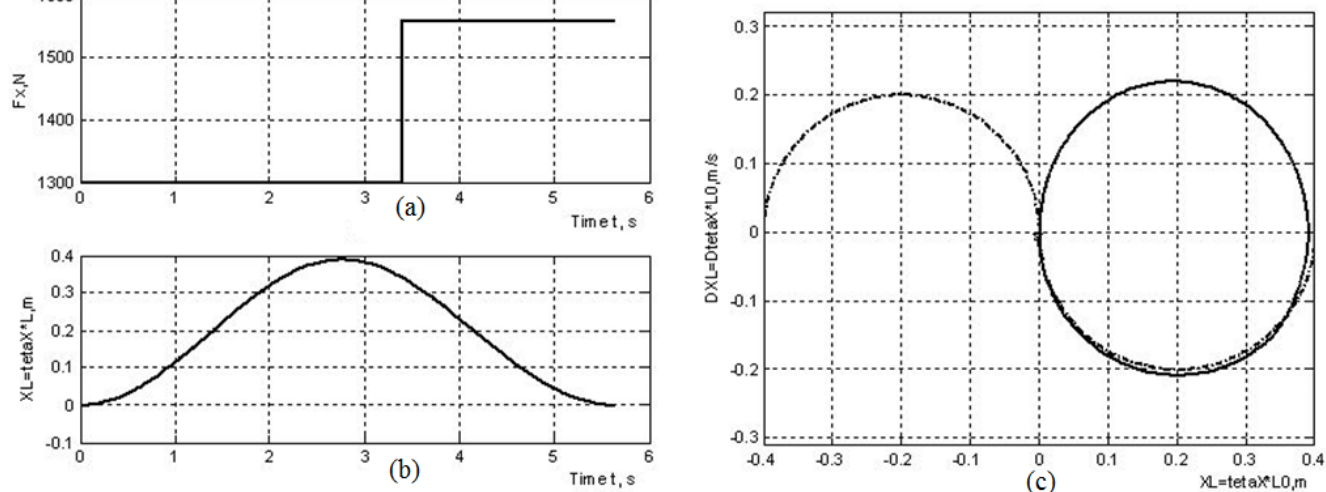

(c)

Fig. 7. Traction force (a) and load oscillation amplitude (b) vs. time plots and phase trajectory plot (c) obtained for the case of the significant deviation of the rope from vertical position at the moment of change of the traction force

\section{REFERENCES}

[1] N. Sun, Y. Fang, "New energy analytical results for the regulation of underactuated overhead cranes: an end-effector motion-based approach", IEEE Transactions on Industrial Electronics, Vol. 59, No. 12, pp. 47234734, 2012

[2] K. C. C. Peng, W. Singhose, D. H. Frakes, "Hand motion crane control using radio-frequency real-time location systems", IEEE/ASME Transactions on Mechatronics, Vol. 17, No. 3, pp. 464-471, 2012

[3] J. Yi, N. Yubazaki, K. Hirota, "Anti-swing and positioning control of overhead traveling crane”, Information Sciences, Vol. 155, No. 1-2, pp. 19-42, 2003

[4] M. Fliess, J. Levine, P. Rouchon, "A simplified approach of crane control via a generalized state-space model", 30th IEEE Conference on Decision and Control, pp. 736-741, 1991

[5] A. J. Ridout, "Anti-swing control of the overhead crane using linear feedback", Australian Journal of Electrical and Electronics Engineering, Vol. 9, No. 1-2, pp. 17-26, 1989

[6] G. G. Parker, B. Petterson, C. Dohrmann, R. D. Robinett, "Command shaping for residual vibration free crane maneuvers", 1995 American Control Conference, pp. 934-938, 1995

[7] B. Vikramaditya, R. Rajamani, "Nonlinear control of a trolley crane system”, 2000 American Control Conference, pp. 1032-1036, 2000

[8] D. T. Liu, J. Q. Yi, D. B. Zhao, "Fuzzy tuning sliding mode control of transporting for an overhead crane", 2003 International Conference on Machine Learning and Cybernetics, pp. 2541-2546, 2003
[9] Y. Fang, W. E. Dixon, D. M. Dawson, E. Zergeroglu, "Nonlinear coupling control laws for a 3-DOF overhead crane system", 40th IEEE Conference on Decision and Control, pp. 3766-3771, 2001

[10] J. Yu, F. L. Lewis, T. Huang, "Nonlinear feedback control of a gantry crane", 1995 American Control Conference, pp. 4310-4315, 1995

[11] J. H. Yang, K. S. Yang, “Adaptive coupling control for overhead crane systems", Mechatronics, Vol. 17, No. 2-3, pp. 143-152, 2007

[12] Y. Al-Sweiti, D. Soffker, "Modeling and control of an elastic shipmounted crane using variable gain model-based controller", Journal of Vibration and Control, Vol. 13, No. 5, pp. 657-685, 2007

[13] J. Huang J, Z. Liang, Q. Zang, "Dynamics and swing control of doublependulum bridge cranes with distributed-mass beams", Mechanical Systems and Signal Processing, Vol. 54-55, pp. 357-366, 2015

[14] S. Lahres, H. Aschemann, O. Sawodny, E. P. Hofer, "Crane automation by decoupling control of a double pendulum using two translational actuators", 2000 American Control Conference, pp. 1052-1056, 2000

[15] Z. N. Masoud, A. H. Nayfeh, N. A. Nayfeh, "Sway reduction on quayside container cranes using delayed feedback controller: simulations and experiments", Journal of Vibration and Control, Vol. 11, No. 8, pp. 1103-1122, 2005

[16] A. H. W. Chun, R. Y. M. Wong, "Improving quality of crane-lorry assignments with constraint programming", IEEE Transactions on Systems, Man, and Cybernetics, Part C (Applications and Reviews), Vol. 37, No. 2, pp. 268-277, 2007 
[17] E. M. Abdel-Rahman, A. H. Nayfeh, Z. N. Masoud, "Dynamics and control of cranes: a review", Journal of Vibration and Control, Vol. 9 , No. 7, pp. 863-908, 2003

[18] W. Singhose, W. Seering, N. Singer, "Residual vibration reduction using vector diagrams to generate shaped inputs", Journal of Mechanical Design, Vol. 116, No. 2, pp. 654-659, 1994

[19] W. Yu, M. A. Moreno-Armendariz, F. O. Rodriguez, "Stable adaptive compensation with fuzzy CMAC for an overhead crane", Information Sciences, Vol. 181, No. 21, pp. 4895-4907, 2011

[20] J. Smoczek, J. Szpytko, "Evolutionary algorithm-based design of a fuzzy TBF predictive model and TSK fuzzy anti-sway crane control system", Engineering Applications of Artificial Intelligence, Vol. 28, pp. 190200,2014

[21] B. Spruogis, A. Jakstas, V. Gican, V. Turla, "Overhead crane anti-swing system based on the Pontryagin's maximum principle", Transport, Vol. 30, No. 1, pp. 61-68, 2015

[22] J. Klosinski, "Swing-free stop control of the slewing motion of a mobile crane", Control Engineering Practice, Vol. 13, No. 4, pp. 451-460, 2005

[23] V. K. Augustaitis, V. Gican, N. Sesok, I. Iljin, "Computer-aided generation of equations and structural diagrams for simulation of linear stationary mechanical dynamic systems", Mechanika, Vol. 17, No. 3, pp. $255-263,2011$

[24] L. S. Pontryagin, V. G. Boltyanskij, R. V. Gamkrelidze, Mathematical theory of optimal processes, Nauka, 1969 